\title{
Proposed imaging of the ultrafast electronic motion in samples using x-ray phase-contrast
}

\author{
Gopal Dixit, ${ }^{1, *}$ Jan Malte Slowik, ${ }^{1,2,+}$ and Robin Santra ${ }^{1,2,+}$ \\ ${ }^{1}$ Center for Free-Electron Laser Science, DESY, \\ Notkestrasse 85, D-22607 Hamburg, Germany \\ ${ }^{2}$ Department of Physics, University of Hamburg, D-20355 Hamburg, Germany
}

(Dated: February 28, 2013)

\begin{abstract}
Tracing the motion of electrons has enormous relevance to understanding ubiquitous phenomena in ultrafast science, such as the dynamical evolution of the electron density during complex chemical and biological processes. Scattering of ultrashort x-ray pulses from an electronic wavepacket would appear to be the most obvious approach to image the electronic motion in real-time and real-space with the notion that such scattering patterns, in the far-field regime, encode the instantaneous electron density of the wavepacket. However, recent results by Dixit et al. [Proc. Natl. Acad. Sci. U.S.A., 109, 11636 (2012)] have put this notion into question and shown that the scattering in the far-field regime probes spatio-temporal density-density correlations. Here, we propose a possible way to image the instantaneous electron density of the wavepacket via ultrafast x-ray phase contrast imaging. Moreover, we show that inelastic scattering processes, which plague ultrafast scattering in the far-field regime, do not contribute in ultrafast x-ray phase contrast imaging as a consequence of an interference effect. We illustrate our general findings by means of a wavepacket that lies in the time and energy range of the dynamics of valence electrons in complex molecular and biological systems. This present work offers a potential to image not only instantaneous snapshots of nonstationary electron dynamics, but also the Laplacian of these snapshots which provide information about the complex bonding and topology of the charge distributions in the systems.
\end{abstract}

PACS numbers: 31.10.+z, 42.50.Ct, 82.53.Hn, 87.15.ht 
In the past, momentum spectroscopy was used to study the internal dynamics of electrons in stationary states [1-3]. According to quantum mechanics, the outcome of measurements on stationary states is time-independent. In contrast, electronic wavepackets are non-stationary states which undergo time-evolution and thus give rise to time-dependent measurements. Therefore, access to the dynamics of non-stationary electrons provides new information about phenomena at the microscopic level. The distinctive timescale of electronic motion, responsible for chemical bonding and electron transfer processes in molecules and complex biological systems, ranges from a few femtoseconds $\left(1 \mathrm{fs}=10^{-15} \mathrm{~s}\right)$ to several attoseconds ( 1 as $=10^{-18} \mathrm{~s}$ ). In order to gain insight into ultrafast chemical or physical processes, one has to unravel the motion of electrons with spatial and temporal resolutions of order $1 \AA$ and 1 fs, respectively [4 6]. Pump-probe experiments are the most direct approach to investigate fast-evolving microscopic processes, where first a pump pulse triggers the dynamics and then subsequently a probe pulse interrogates such triggered dynamics as a function of pump-probe delay time. In the last few years, with the availability of laser pulses on the sub-fs timescale [7, 8], remarkable progress has been made towards the understanding of electronic motion in real time [9-15]. In recent years, due to advancement in technology, it has become possible to generate ultraintense, ultrashort and tunable x-ray pulses from novel light sources such as free-electron lasers (FEL) [16, 17], laser plasmas [18] and highharmonic generation [19, 20], which may provide a unique opportunity to investigate these ultrafast processes with atomic-scale spatial and temporal resolution. Since the beginning of the operation of the first FEL in the hard x-ray regime, the Linac Coherent Light Source, high-intensity x-ray experiments have been carried out for systems ranging from atoms [21], small molecules [22], complex biomolecules [23], to matter in extreme conditions [24].

To image electronic motion in real-time and real-space, which is important to understand several ultrafast complex processes, one can perform far-field scattering of ultrashort x-ray pulses from the dynamically evolving electronic system. By varying the pump-probe time delay one obtaines a series of scattering patterns that serve to image the electronic motion with atomic-scale spatio-temporal resolution. Based on experience with elastic scattering from electronically stationary targets, one might expect to be able to retrieve the instantaneous electron density (IED) of the electronic wavepacket via ultrafast scattering in far-field regime. However, for probing the motion of the wavepacket on an ultrafast timescale, one needs an ultrashort pulse with unavoidable bandwidth. Due to the inherent bandwidth, 
there is no way to know whether the probe pulse induces transitions among the eigenstates spanning the wavepacket, or to other states closer in energy than the bandwidth. As a result, the probe pulse inevitably changes the wavepacket and the pattern contains contributions from all the states within the bandwidth, that were accessed during the scattering process. Therefore, it is impossible to transfer the concepts underlying stationary coherent scattering to the ultrafast regime. Recently, Dixit et al. [25] have investigated ultrafast scattering from an electronic wavepacket in the far-field regime and shown that the scattering patterns encode spatial and temporal correlations that deviate completely from the notion of the IED as the key quantity being probed [4, 26]. Moreover, they have shown that, ultimately this is a consequence of quantum electrodynamics and can not be captured semiclassically [25]. Therefore, it is not possible to retrieve direct information about the dynamical structural changes during the complex processes from these correlations. On the other hand, if one can retrieve the IED at different instants of time during the processes, it will provide direct insight about these processes. In light of this, one may wonder if there is any way, using light, to image the IED of the wavepacket in real-space at different instants of time during the motion of the wavepacket.

In this Letter, we demonstrate rigorously how the IED of an electronic wavepacket can be imaged via ultrafast phase contrast imaging (PCI). The two important classes of imaging are near-field imaging and far-field imaging (see Fig. 1) [27, 28]. In the following, we discuss the pros and cons of imaging the motion of the wavepacket in both regimes. In far-field imaging, one only detects the scattered radiation and obtains the Fourier space image of the object from which the scattering has taken place. This is what is normally done in x-ray crystallography [27]. On the other hand, in near-field imaging, one detects the interference between incident and scattered radiation [27]. X-ray PCI falls into the near-field imaging regime, with many applications in various fields of science including biology [29, 30] and condensed matter physics [31, 32]. Photoelectron holography [33 35] is an alternative approach to imaging electronic motion. We expect that the technique of ultrafast x-ray PCI proposed here will be particularly useful for studying spatially extended systems, which are often inaccessible to methods based on photoelectrons.

We apply a consistent quantum theory for the radiation field and the electrons, by treating both using the language of quantum field theory. Following from the principle of minimal coupling in Coulomb gauge, the Hamiltonian describing the interaction between radiation 


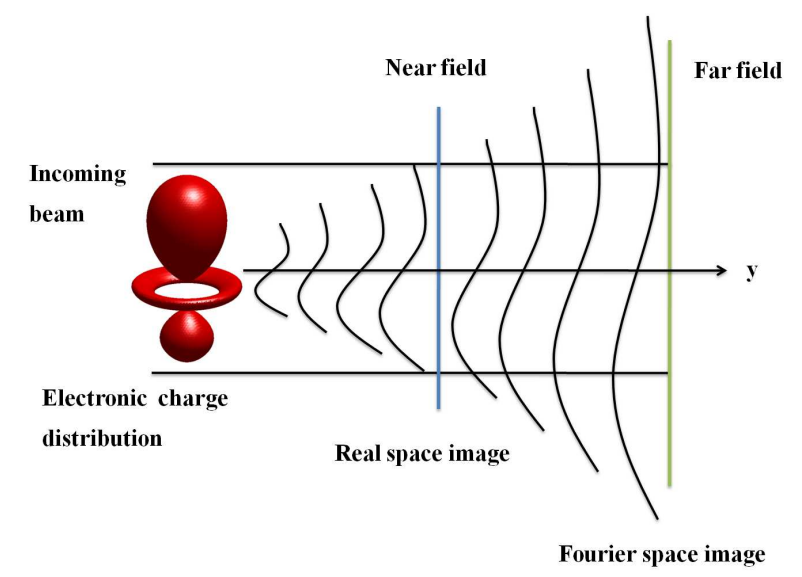

FIG. 1. (color online). Concept of radiation-matter interaction, where matter is represented by an isosurface of an electronic charge distribution of an electronic wavepacket. The real-space phasecontrast image is observed in the near-field region, whereas the Fourier-space scattering pattern is observed in the far-field region.

field and electrons is [36]

$$
\begin{aligned}
\hat{H}_{\mathrm{int}}= & -\frac{e}{m} \int d^{3} x \hat{\psi}^{\dagger}(\mathbf{x})\left[\hat{\mathbf{A}}(\mathbf{x}) \cdot \frac{\hbar}{i} \nabla\right] \hat{\psi}(\mathbf{x}) \\
& +\frac{e^{2}}{2 m} \int d^{3} x \hat{\psi}^{\dagger}(\mathbf{x}) \hat{\mathbf{A}}^{2}(\mathbf{x}) \hat{\psi}(\mathbf{x}) .
\end{aligned}
$$

Here, the field operator $\hat{\psi}^{\dagger}(\mathbf{x})[\hat{\psi}(\mathbf{x})]$ creates (annihilates) an electron at position $\mathbf{x} ; e$ and $m$ are charge and mass of the electron, respectively; $(\hbar / i) \nabla$ represents the canonical momentum of an electron, $\hbar$ being the reduced Planck constant; and $\hat{\mathbf{A}}$ is the vector potential operator of the radiation field. Here, we only focus on scattering events induced by the $\hat{\mathbf{A}}^{2}$ operator and will not consider the contribution from the $\hat{\mathbf{A}}(\mathbf{x}) \cdot \nabla$ term in Eq. (1) in the scattering process as it is known that its contribution is negligibly small at photon energies much higher than all inner-shell thresholds in the system of interest [27]. However, it is useful to mention that the scattering induced by the $\hat{\mathbf{A}}(\mathbf{x}) \cdot \nabla$ term in second order gives rise to the KramersHeisenberg cross section, which describes the powerful photon-in/photon-out technique of resonant inelastic x-ray scattering [37-39]. Note that inelastic and elastic scattering at high photon energy, i.e., Compton and Thomson scattering, is mediated by the $\hat{\mathbf{A}}^{2}$ operator. Since we are working in a particular gauge (the Coulomb gauge), our approach is not gauge invariant.

Let us assume that with the help of a pump pulse, one prepares a coherent superpo- 
sition of eigenstates (electronic wavepacket) with $\hat{\rho}_{\text {in }}^{\text {el }}=\left|\Psi_{\text {in }}\right\rangle\left\langle\Psi_{\text {in }}\right|$ as the initial density operator for the wavepacket. The multimode radiation field is treated as a collection of photons occupying different electromagnetic modes with $\hat{\rho}_{\text {in }}^{X}$ as the initial density operator of the field [40]. Before the interaction takes place, the entire system is therefore prepared in the initial state $\hat{\rho}_{\text {in }}=\hat{\rho}_{\text {in }}^{X} \otimes \hat{\rho}_{\text {in }}^{\text {el }}$. The density operator evolves according to $\hat{\rho}(t)=\lim _{t_{0} \rightarrow-\infty} \hat{U}_{I}\left(t, t_{0}\right) \hat{\rho}_{\text {in }} \hat{U}_{I}^{\dagger}\left(t, t_{0}\right)$, with the time-evolution operator $\hat{U}_{I}$ satisfying the equation of motion with respect to $\hat{H}_{\text {int }}$ in the interaction picture. Making a perturbation expansion of the time evolution operator to first order with respect to $\hat{H}_{\text {int }}$, the expectation value of an observable $\hat{\mathcal{O}}$ at time $t$ is expressed as

$$
\begin{aligned}
\langle\hat{\mathcal{O}}\rangle_{t}= & \operatorname{Tr}(\hat{\rho}(t) \hat{\mathcal{O}}(t)) \\
= & \operatorname{Tr}\left(\hat{\rho}_{\text {in }} \hat{\mathcal{O}}(t)\right)+2 \operatorname{Re}\left\{\frac{i}{\hbar} \int_{-\infty}^{t} d t^{\prime} \operatorname{Tr}\left(\hat{\rho}_{\text {in }} \hat{H}_{\text {int }}\left(t^{\prime}\right) \hat{\mathcal{O}}(t)\right)\right\} \\
& +\frac{1}{\hbar^{2}} \int_{-\infty}^{t} \int_{-\infty}^{t} d t^{\prime} d t^{\prime \prime} \operatorname{Tr}\left(\hat{H}_{\text {int }}\left(t^{\prime \prime}\right) \hat{\rho}_{\text {in }} \hat{H}_{\text {int }}\left(t^{\prime}\right) \hat{\mathcal{O}}(t)\right) .
\end{aligned}
$$

Here, $\hat{\mathcal{O}}$ evolves with respect to $\hat{H}_{0}=\hat{H}_{\text {el }}+\hat{H}_{\text {rad }}$, i.e., $\hat{\mathcal{O}}(t)=\exp \left(\frac{i}{\hbar} \hat{H}_{0} t\right) \hat{\mathcal{O}} \exp \left(-\frac{i}{\hbar} \hat{H}_{0} t\right)$, where $\hat{H}_{\mathrm{el}}$ and $\hat{H}_{\text {rad }}$ are the Hamiltonians corresponding to the electrons and the radiation field, respectively. If the observable is the intensity at the detector, the interpretation of the terms on the right-hand side of Eq. (2) is straightforward. The first term describes the intensity of the incident radiation; the second term describes the interference between the incident and the scattered radiation; and the last term represents the scattered radiation only (see Fig. 1).

Now we focus on those terms that are responsible for ultrafast PCI, i.e., the first and second terms on the right-hand side of Eq. (2). Modelling the intensity observable at the detector $\hat{\mathcal{O}}$ by the Poynting operator that acts only on the radiation part [41], the state of the electronic system after the scattering event remains undetected. Thus the second term can be written as

$$
\begin{aligned}
\operatorname{Tr}\left(\hat{\rho}_{\text {in }} \hat{H}_{\text {int }}\left(t^{\prime}\right) \hat{\mathcal{O}}(t)\right)= & \frac{e^{2}}{2 m} \int d^{3} x \operatorname{Tr}_{X}\left(\hat{\rho}_{\text {in }}^{X} \hat{\mathbf{A}}^{2}\left(\mathbf{x}, t^{\prime}\right) \hat{\mathcal{O}}(t)\right) \\
& \times \operatorname{Tr}_{\text {el }}\left(\hat{\rho}_{\text {in }}^{\text {el }} \hat{n}\left(\mathbf{x}, t^{\prime}\right)\right)
\end{aligned}
$$

where $\operatorname{Tr}_{X}$ and $\operatorname{Tr}_{\text {el }}$ are the traces over radiation and electrons, respectively; and $\hat{n}(\mathbf{x})=$ $\hat{\psi}^{\dagger}(\mathbf{x}) \hat{\psi}(\mathbf{x})$ is the electron density operator. In this case,

$$
\operatorname{Tr}_{\text {el }}\left(\hat{\rho}_{\text {in }}^{\mathrm{el}} \hat{n}\left(\mathbf{x}, t^{\prime}\right)\right)=\left\langle\Psi_{\text {in }}\left|\hat{n}\left(\mathbf{x}, t^{\prime}\right)\right| \Psi_{\text {in }}\right\rangle=\rho\left(\mathbf{x}, t^{\prime}\right)
$$


Here, $\rho\left(\mathbf{x}, t^{\prime}\right)$ is the IED of the wavepacket at time $t^{\prime}$. At this point, it is interesting to note that the probe pulse inevitably changes the wavepacket. But due to the interference between the incident and scattered radiation, described by Eq. (3), eigenstates outside the wavepacket cannot contribute to the signal at the detector in the case of near-field imaging [see Eq. (4)]. This present result is in contrast to the results obtained in the case of far-field imaging, where one only observes scattered radiation. An analysis of the third term on the right-hand side of Eq. (2) demonstrates that the patterns obtained via far-field imaging contain the fingerprint of electronic transitions within the bandwidth of the probe pulse [25].

Now, the x-ray pulse is considered to be quasi-stationary, quasi-monochromatic, and spatially coherent. Furthermore, we assume that the x-ray pulse duration is sufficiently short to freeze the dynamics of the wavepacket, and the pulse propagates along the $y$ axis. Therefore, using quantum theory as shown in the case of x-ray PCI from a stationary sample [41], we find that the time-resolved phase contrast image is related to the Laplacian of the projected instantaneous electron density, $\rho_{\perp}\left(\mathbf{r}_{\perp}, \tau\right)=\int d r_{y} \rho(\mathbf{r}, \tau)$ with $\mathbf{r}=\left(r_{x}, r_{y}, r_{z}\right), \mathbf{r}_{\perp}=\left(r_{x}, r_{z}\right)$ and $\tau$ is the pump-probe delay time. Therefore, the signal at the detector in the case of ultrafast PCI is

$$
I(\mathbf{r})=I_{\text {in }}\left(1-\frac{2 \pi r_{e} D}{\left|\mathbf{k}_{\text {in }}\right|^{2}} \nabla^{2} \rho_{\perp}\left(\mathbf{r}_{\perp}, \tau\right)\right),
$$

where $I_{\text {in }}$ is the signal due to the incident radiation alone, $\mathbf{k}_{\text {in }}$ is the wavevector of the incident radiation, $r_{e}$ is the classical electron radius, and $D$ is the distance of the detector from the object. The key quantity in ultrafast PCI is the Laplacian of the projected IED of the wavepacket. The experimental parameters for performing ultrafast PCI are subject to certain conditions. The near-field regime is in general characterized by a Fresnel number $F \gtrsim 1$, which relates the spatial extension $a$ of the wavepacket with $D$ and the wavelength $\lambda$ of the incident radiation via $F=\frac{a^{2}}{\lambda D}$. Quasi-monochromaticity of the pulse is ensured by a sufficiently long coherence time; however, $\tau_{\text {tc }}$ has to be short with respect to the propagation time of the radiation to the detector, $\frac{\lambda}{2 \pi c} \ll \tau_{\text {tc }} \ll \frac{D}{c}$, c denoting the speed of light.

The two-dimensional (2D) projection of the IED, $\rho_{\perp}$, can be retrieved by applying a suitable Poisson solver algorithm to a single recorded image. The full three-dimensional (3D) tomographic distribution of the IED can be reconstructed by recording several 2D projected images for many different projection angles. Several tomographic methods to reconstruct the 3D distribution have been developed, as reviewed by Burvall et al. [42]. A direct method for reconstructing the 3D IED, without intermediate reconstruction of the 2D 
projected IED $\rho_{\perp}$, was presented in Ref. [43]. By taking images of the object rotated around the $z$ axis about an angle $\theta$, described by the density $\rho_{\theta}$, one obtains the data sets $g_{\theta}=\nabla^{2} \rho_{\theta \perp}$ from Eq. (51). The reconstruction algorithm has the form of a filtered backprojection. By the Fourier projection theorem [44] the Fourier transform of a data function $g_{\theta}$ corresponds to a rotated plane in Fourier space of the 3D object. The 3D object can be reconstructed by applying the filter function $Q\left(k_{x_{\theta}}, k_{z}\right)=\left|k_{x_{\theta}}\right| /\left(k_{x_{\theta}}^{2}+k_{z}^{2}\right)$ in Fourier space and integrating over all rotation angles,

$$
\rho(\mathbf{r}, \tau)=\frac{1}{4 \pi^{2}} \int_{0}^{\pi} d \theta \mathcal{F}^{-1}\left[Q \mathcal{F}\left[g_{\theta}\right]\right]\left(x_{\theta}, z\right),
$$

where $x_{\theta}=x \cos \theta+y \sin \theta$ and $\mathcal{F}$ denotes the 2D Fourier transform. The 3D Laplacian of the IED, $\nabla^{2} \rho(\mathbf{r}, \tau)$, can be obtained by applying the filter $Q\left(k_{x_{\theta}}, k_{z}\right)=\left|k_{x_{\theta}}\right|$ in the filtered backprojection. Therefore, ultrafast PCI provides the full 3D Laplacian of the IED, which highlights internal and external boundaries of the wavepacket e.g., zero flux boundaries - a very useful and informative quantity according to Bader's theory of atoms in molecules, where the Laplacian of the electronic density is used to provide detailed information about the complex bonding and topology of the charge distributions in molecules [45, 46].

In order to illustrate the generality of our proposed method for imaging the IED of the wavepacket, we apply ultrafast PCI to the same electronic wavepacket as was considered by Dixit et al. [25]. In this case, the electronic wavepacket is prepared as a coherent superposition of the $3 d$ and $4 f$ eigenstates of atomic hydrogen, each eigenstate having a population of $50 \%$ and the projection of orbital angular momentum being equal to zero. The oscillation period of the wavepacket is $\mathrm{T}=6.25 \mathrm{fs}$, which is inversely related to the energy spacing between the $3 d$ and $4 f$ eigenstates $(0.66 \mathrm{eV})$. It is important to emphasize that the considered wavepacket lies in the energy and timescale of the valence electronic motion in more complex molecular and biological systems [47-50]. Moreover, in many-electron systems, where only few electrons get excited and participate in the formation of an electronic wavepacket, the electronic density of the system can be decomposed into a time-dependent density of excited electrons and a static density of the stationary electrons.

Figure 2(a) shows phase contrast images calculated with the Laplacian of the projected IED, $\nabla^{2} \rho_{\perp}$ as a function of the delay time at times $0, \mathrm{~T} / 4, \mathrm{~T} / 2,3 \mathrm{~T} / 4$, and T. Figure 2 (b) shows the projected IED, $\rho_{\perp}$, of the wavepacket, where the electronic charge distribution periodically undergoes localization to delocalization from positive to negative $z$ axis within 
(a)
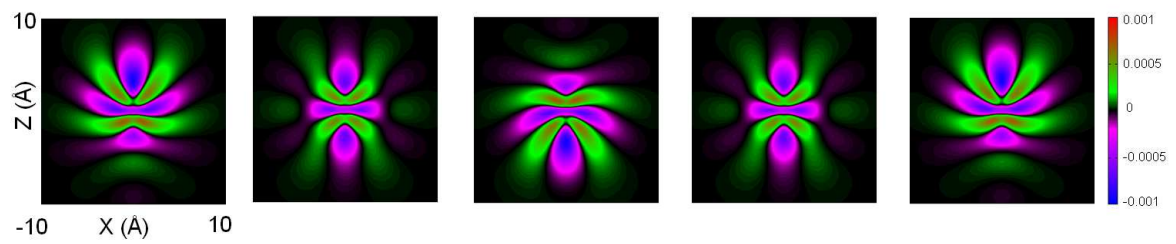

0

$\mathrm{T} / 4$

$\mathrm{T} / 2$

$3 \mathrm{~T} / 4$

T

(b)
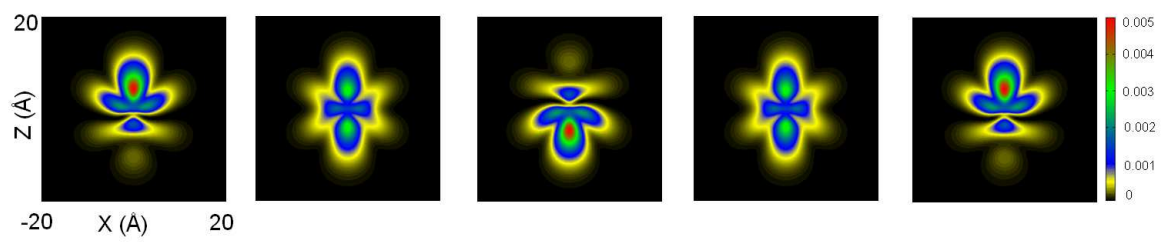

FIG. 2. (color online). Phase contrast images and projected instantaneous electron density of the wavepacket. (a) Laplacian of the projected instantaneous electron density, and (b) the instantaneous electron density integrated along the direction of propagation of the incident $\mathrm{x}$ rays ( $y$ axis) at pump-probe delay times $0, \mathrm{~T} / 4, \mathrm{~T} / 2,3 \mathrm{~T} / 4$, and $\mathrm{T}$, where the oscillation period of the electronic wavepacket is $\mathrm{T}=6.25 \mathrm{fs}$.

6.25 fs. The spatial extension of $\rho_{\perp}$ is $14-17 \AA$ along the $z$ axis and $7.5-9 \AA$ along the $x$ axis. As the electronic charge distribution oscillates from positive to negative $z$ axis and returns back to its original position, the patterns obtained by ultrafast PCI follow the same trend of the oscillation and provide the correct time period of the oscillation. At delay times $\mathrm{T} / 4$ and $3 \mathrm{~T} / 4$, the charge distributions are completely delocalized and identical to each other, and the phase contrast images are also identical (see Fig. 2). The present results are fundamentally different from the scattering patterns obtained via far-field scattering, where the patterns are completely different at delay times $\mathrm{T} / 4$ and $3 \mathrm{~T} / 4$, whereas the electronic charge distributions are identical at those times [25]. Similarly, the charge distributions are completely different at times 0 and $\mathrm{T} / 2$, which is also reflected in the phase contrast images at those delay times, whereas the far-field scattering patterns are identical at times 0 and $\mathrm{T} / 2$ [25]. Clearly, the images obtained with ultrafast PCI are directly related to the IED of the wavepacket.

The patterns obtained via ultrafast PCI are rich in information. Figure 2(a) shows more structures than the corresponding $\rho_{\perp}$ shown in Fig. 2(b). It is important to notice that the images have both positive and negative lobes, whereas the densities have only positive lobes. 
The $3 d$ and $4 f$ eigenstates in the wavepacket have no radial nodes, they only have angular nodes. The PCI images show the curvature of these angular nodes in the wavepacket. The electronic charge distribution is locally concentrated where $\nabla^{2} \rho_{\perp}<0$ and depleted where $\nabla^{2} \rho_{\perp}>0$. Therefore, these images provide the topology of the electronic charge distribution with detailed information about the nodal structure of the wavepacket.

In conclusion, we have demonstrated that the IED of the wavepacket can be imaged via ultrafast PCI. Therefore, ultrafast PCI can provide direct information about the dynamical changes in the spatial electron probability distribution at different instants of time. Morever, ultrafast PCI provides the Laplacian of the IED, which reveals the internal structures of the wavepacket through local variations in the IED. Ultrafast PCI does not rely on any assumptions such as the single-active-electron approximation [51, 52]. The technique is therefore applicable to large and strongly correlated systems and ultrafast PCI would be very powerful for imaging electronic quantum motion in real-time and real-space with potentially unprecedented spatio-temporal resolution. Ultrafast PCI may be expected to shed light on non-equilibrium quantum motion, for example, in peptides and biological systems [47-49]. Therefore, with the tremendous technological advancement in x-ray imaging technologies [53, 54], one might expect ultrafast PCI measurements to be feasible in the future.

We thank Sang-Kil Son for careful reading of the manuscript.

* gopal.dixit@cfel.de

$\dagger$ jan.malte.slowik@cfel.de

$\ddagger$ robin.santra@cfel.de

[1] M. J. Cooper, Rep. Prog. Phys. 48, 415 (1999).

[2] I. E. McCarthy and E. Weigold, Rep. Prog. Phys. 54, 789 (1999).

[3] C. E. Brion, G. Cooper, Y. Zheng, I. V. Litvinyuk, and I. E. McCarthy, Chemical Physics 270, 13 (2001).

[4] F. Krausz and M. Ivanov, Rev. Mod. Phys. 81, 163 (2009).

[5] P. H. Bucksbaum, Science 317, 766 (2007).

[6] P. B. Corkum and F. Krausz, Nature Physics 3, 381 (2007).

[7] E. Goulielmakis et al., Science 320, 1614 (2008). 
[8] M. Hentschel et al., Nature 414, 509 (2001).

[9] S. Haessler et al., Nature Physics 6, 200 (2010).

[10] P. Tzallas, E. Skantzakis, L. A. A. Nikolopoulos, G. D. Tsakiris, and D. Charalambidis, Nature Physics 7, 781 (2011).

[11] P. Hockett, C. Z. Bisgaard, O. J. Clarkin, and A. Stolow, Nature Physics 7, 612 (2011).

[12] H. Niikura et al., Nature 421, 826 (2002).

[13] E. Goulielmakis et al., Nature 466, 739 (2010).

[14] G. Sansone et al., Nature 465, 763 (2010).

[15] O. Smirnova, Y. Mairesse, S. Patchkovskii, N. Dudovich, D. Villeneuve, P. Corkum, and M. Y. Ivanov, Nature 460, 972 (2009).

[16] P. Emma et al., Nature Photonics 4, 641 (2010).

[17] T. Ishikawa et al., Nature Photonics 6, 540 (2012).

[18] A. Rousse, C. Rischel, and J. C. Gauthier, Rev. Mod. Phys. 73, 17 (2001).

[19] I. McKinnie and H. Kapteyn, Nature Photonics 4, 149 (2010).

[20] T. Popmintchev, M. C. Chen, D. Popmintchev, P. Arpin, S. Brown, S. Ališauskas, G. Andriukaitis, T. Balčiunas, O. D. Mücke, A. Pugzlys, A. Baltuska, B. Shim, S. E. Schrauth, A. Gaeta, C. Hernandez-Garcia, L. Plaja, A. Becker, A. Jaron-Becker, M. M. Murnane, and H. C. Kapteyn, Science 336, 1287 (2012).

[21] L. Young et al., Nature 466, 56 (2010).

[22] N. Berrah et al., Proc. Natl. Acad. Sci. U.S.A 108, 16912 (2011).

[23] H. N. Chapman et al., Nature 470, 73 (2011).

[24] S. M. Vinko et al., Nature 482, 59 (2012).

[25] G. Dixit, O. Vendrell, and R. Santra, Proc. Natl. Acad. Sci. U.S.A 109, 11636 (2012).

[26] H. C. Shao and A. F. Starace, Phys. Rev. Lett. 105, 263201 (2010).

[27] J. Als-Nielsen and D. McMorrow, Elements of modern X-ray physics, Wiley, New York, 2011.

[28] K. A. Nugent, Adv. Phys. 59, 1 (2010).

[29] T. J. Davis, D. Gao, T. E. Gureyev, A. W. Stevenson, and S. W. Wilkins, Nature 373, 595 (1995).

[30] S. W. Wilkins, T. E. Gureyev, D. Gao, A. Pogany, and A. W. Stevenson, Nature 384, 335 (1996).

[31] T. E. Gureyev, S. Mayo, S. W. Wilkins, D. Paganin, and A. W. Stevenson, Phys. Rev. Lett. 
86, 5827 (2001).

[32] M. D. De Jonge, B. Hornberger, C. Holzner, D. Legnini, D. Paterson, I. McNulty, C. Jacobsen, and S. Vogt, Phys. Rev. Lett. 100, 163902 (2008).

[33] S. Chelkowski, G. L. Yudin, and A. D. Bandrauk, Journal of Physics B 39, S409 (2006).

[34] X. B. Bian and A. D. Bandrauk, Phys. Rev. Lett. 108, 263003 (2012).

[35] Y. Huismans, A. Rouzée, A. Gijsbertsen, J. H. Jungmann, A. S. Smolkowska, P. S. W. M. Logman, F. Lepine, C. Cauchy, S. Zamith, T. Marchenko, J. M. Bakker, G. Berden, B. Redlich, A. F. G. van der Meer, H. G. Muller, W. Vermin, K. J. Schafer, M. Spanner, M. Y. Ivanov, O. Smirnova, D. Bauer, S. V. Popruzhenko, and M. J. J. Vrakking, Science 331, 61 (2011).

[36] D. P. Craig and T. Thirunamachandran, Molecular Quantum Electrodynamics, Academic Press, London, 1984.

[37] Y. Ma, Phys. Rev. B 49, 5799 (1994).

[38] J. D. Mills, J. A. Sheehy, T. A. Ferrett, S. H. Southworth, R. Mayer, D. W. Lindle, and P. W. Langhoff, Phys. Rev. Lett. 79, 383 (1997).

[39] W. Schülke, Electron dynamics by inelastic X-ray scattering, Oxford University Press Oxford, UK, 2007.

[40] L. Mandel and E. Wolf, Optical Coherence and Quantum Optics, Cambridge University Press, Cambridge, 1995.

[41] J. M. Slowik and R. Santra, (to be published).

[42] A. Burvall, U. Lundström, P. A. C. Takman, D. H. Larsson, and H. M. Hertz, Opt. Express 19, 10359 (2011).

[43] A. V. Bronnikov, J. Opt. Soc. Am. A 19, 472 (2002).

[44] A. Kak and M. Slaney, Principles of computerized tomographic imaging, Classics in applied mathematics, Society for Industrial and Applied Mathematics, 2001.

[45] R. F. W. Bader and T. T. Nguyen-Dang, Adv. Quantum Chem. 14, 63 (1981).

[46] R. F. Bader, Chem. Rev. 91, 893 (1991).

[47] J. Breidbach and L. S. Cederbaum, J. Chem. Phys. 118, 3983 (2003).

[48] A. I. Kuleff, J. Breidbach, and L. S. Cederbaum, J. Chem. Phys. 123, 044111 (2005).

[49] F. Remacle and R. D. Levine, Proc. Natl. Acad. Sci. U.S.A 103, 6793 (2006).

[50] G. D. Scholes, G. R. Fleming, A. Olaya-Castro, and R. van Grondelle, Nature Chemistry 3, $763(2011)$. 
[51] J. Itatani, J. Levesque, D. Zeidler, H. Niikura, H. Pépin, J. C. Kieffer, P. B. Corkum, and D. M. Villeneuve, Nature 432, 867 (2004).

[52] C. I. Blaga, J. Xu, A. D. DiChiara, E. Sistrunk, K. Zhang, P. Agostini, T. A. Miller, L. F. DiMauro, and C. D. Lin, Nature 483, 194 (2012).

[53] A. Sakdinawat and D. Attwood, Nature Photonics 4, 840 (2010).

[54] S. M. Gruner, Phys. Today 65, 29 (2012). 